\title{
Supporting Remote Creative Collaboration in Film Scoring
}

\author{
Julien Phalip ${ }^{1,2,3}$, Ernest A. Edmonds ${ }^{1,2}$, David Jean ${ }^{4}$ \\ julien@julienphalip.com, ernest@ernestedmonds.com, jeandavid31@gmail.com \\ ${ }^{1}$ Creativity and \\ ${ }^{2}$ Australasian CRC \\ for Interaction Design \\ Australia

$\begin{array}{cc}{ }^{3} \text { IREMIA } & { }^{4} \text { University of } \\ \text { University of } & \text { Toulouse } \\ \text { La Réunion } & \text { France }\end{array}$ \\ Cognition Studios University of \\ Technology, Sydney Australia \\ France
}

\begin{abstract}
This paper reports on research supporting online and asynchronous collaboration between stakeholders in the film scoring industry. Here, the authors present two studies conducted with filmmakers and composers to test the design principles of a prototype system. Outcomes from this research have identified a need for establishing a clear scope in creative discussions and for resolving the ambiguity that occurs in remote collaboration. Feedback from participants also revealed the complex nature of the composer-filmmaker relationship and highlighted some interpersonal and coordination issues that should be addressed when designing systems for distant communication.
\end{abstract}

\section{Author Keywords}

Film scoring, creative collaboration, multidisciplinary collaboration, remote communication.

ACM Classification Keywords

H.5.5 Sound and Music Computing; H.5.3 Group and Organization Interfaces

\section{General Terms}

Design, Experimentation

\section{INTRODUCTION}

Amongst multidisciplinary and creative disciplines, film scoring is a complex case that combines significant collaboration characteristics. First, it is based on a clientcommissionee relationship; the clients, namely an individual or a group of filmmakers (directors or producers), start by commissioning a composer to write the music for their film. Second, the clients themselves can actively contribute to the creation of the work; whether or not they are musically literate, filmmakers often give specifications or a set of constraints to the composer so that the music eventually serves their vision. Third, although they have to trust the composer, filmmakers follow the progress of the work to ensure that it takes the right direction and that it will be delivered on time for the film's release. When a creative collaboration is engaged, the composer regularly provides drafts or works in progress to the filmmakers who, in return, provide feedback either to validate the work or to ask for alterations. This process is usually appreciated by all parties as it is a source of creative stimulation and ensures that the work remains concordant with the clients' will and expectations. However, previous qualitative field studies [13] [14], conducted by the authors and based on interviews and observations with 14 filmmakers and 13 composers, have revealed a set of communication challenges commonly faced in this collaborative process. It was found that collaboration can be frustrating and compromising because practitioners do not share the same musical language. This lack of common language causes communication breakdowns due to the exchange of ambiguous, incomplete or inaccurate information. Importantly, it was also observed that a growing number of collaborations are conducted remotely, a situation which can aggravate communication challenges and dramatically affect creative outcomes.

In this paper, we first explain in more detail the problematic conditions of remote communication between stakeholders of the film scoring industry. After reviewing related work, we describe the low-fidelity prototype designed to facilitate the establishment of a clear scope for creative discussions in online and asynchronous settings. We then describe the initial findings from a design study conducted with two composers and a filmmaker to test the appropriateness of the prototype's concepts. Finally, we present the results from the evaluation study of a high-fidelity version of the prototype and close by reflecting further on the issues at stake.

\section{THE PROBLEM}

Most of today's composers and filmmakers are familiar with technology. They have long used electronic and digital tools to facilitate technical tasks such as editing, applying sound and video effects or making mock-ups. Practitioners make use of tools like samplers, sequencers, synthesizers or virtual instruments to extend their composition capabilities and to improve their productivity. The increasing affordability of new technologies also profoundly changes the landscape of the film scoring industry. The practice becomes democratised, as many amateur or aspiring composers now build their own home studios and offer their services.

Since the advent of Internet and fast bandwidths facilitating the exchange of heavy media such as video and music, it has also become commonplace for film composers to work with 
filmmakers remotely located, in different cities or even countries. Although this greatly widens the market and brings new opportunities for collaboration, communication now faces unpredicted challenges. In some instances telephone, regular mail or email communications are sufficient to assist the exchange of music and video footage. However, due to the absence of face-to-face interaction and the inability of current accessible technology to compensate for this absence [16], if problems occur, these problems can be amplified because interactions between people are drastically limited. One consequence of this situation is that practitioners can inadvertently lose track of the conversation's topic. They might not realize, for example, when a mistake is made or when the focus of the conversation deviates. Notably, the lack of visual information (e.g. in phone conversations) or the delays occurring between people's responses (e.g. in email conversations) can introduce indexical dysfunctions that hinder the understanding of what is referred to. In this context, the term 'indexical' pertains to the character of indicating, showing, or pointing at things. It was observed through our field studies that practitioners sometimes experience difficulties in precisely framing the scope of their discussions. If the scope is imprecise or out of phase, the relevance of the conveyed information may be affected, which may in turn diminish the efficiency of communication. This suggests that the problem of ambiguity should be differentiated into two categories: the ambiguity of content - what is said - and the ambiguity of scope - what is talked about. The former is the type of ambiguity which is concerned with the use of vague terms having different meanings or interpretations; for example: "I want some happy music" or "I would like it to sound like the calm before the storm". The latter is the type of ambiguity introduced when one imprecisely or wrongly refers to specific parts of the film. An example was provided by an interviewed composer:

"Once, a director asked me to change the music in a scene with a close-up on a character. But she didn't realise that there were several close-ups of that same character in the film. Unfortunately I changed the music in the wrong place, and then she wondered: 'But, nothing has changed where I told you!'”

Similarly, it can be challenging for the composer to explain in words where to lay the music drafts as they are sent along to the filmmakers; as illustrated by these snippets extracted from various email discussions between composers and directors that we collected through our field studies:

"Please lay it up to start under the high shot of the white building after the team is looking at the map together, then it goes into the cars, then markets, then travel and through to onion lady. Should stop hard just before she says 'OK!’”

“This cue starts during laughing after 'from 5 to 12!' It is hard to precise the start time but there is a gap in the drums as $\mathrm{K}$ says 'it's quite salubrious' and then it stops hard on cut to yukky loo. [...] Hope you are comprehending my rather haphazard instructions about where to lay in all these cues. Call me if you need help with sync.”

"This one goes in the section on Caitlin's work around 24 mins in the cut I have. It starts after she says 'toxic to the plant' on the cut to the wide shot of her in the field. Let it run through silence until the second part appears.”

Unfortunately, mentioning the specific time $\operatorname{code}^{1}$ is not always relevant, as the parties may be working in parallel on different versions of the picture. Indeed, the filmmakers may still be making slight, sometimes even extensive, alterations in the editing room while the composer is writing the music.

There are different viewpoints about whether or not ambiguity benefits design and art practices. On the one hand, Gaver et al. [9] see ambiguity as a virtue which should be embraced. For them, products which provide little context, evoke unfamiliar objects, distort information, have uncertain purpose, or blur lines between reality and virtuality, are more likely to be appealing and engaging because consumers can appropriate them through their own meaning interpretation. On the other hand, Stacey \& Eckert [17] refute the "myth of beneficial ambiguity" in design communication; as they put it, "[c]ommunicating imprecise, uncertain and provisional ideas is a vital part of design teamwork, but what is uncertain and provisional needs to be expressed as clearly as possible”. Indeed, while they admit ambiguity sometimes leads to unexpected and useful discoveries, they also argue that, in practice, ambiguity in design and art conversations much more frequently causes useless and counterproductive misunderstandings. We elaborate on this reasoning by emphasizing priorities of concern between the ambiguity of content and the ambiguity of scope. While we have shown that ambiguity of content is present throughout the filmmaker-composer collaboration [14] we now also argue that, when collaboration is conducted remotely, it is necessary to first resolve the ambiguity of scope by addressing the indexical dysfunctions in communication.

\section{RELATED WORK}

Over recent years creative collaboration and multidisciplinary collaboration have been themes of increasing interest across industrial and academic milieus. Following extensive technological advances in the past decade, a large number of research endeavors have been concerned with the design of tools to provide relief and support in such complex collaborative situations. For example, Bødker et al. [4] have focused on ways and means of stimulating idea generation in cooperative, iterative design. Another example was with Bennett and Dziekan [3] who have explored the concepts of Online Creative Collaboration (OCC) through the Omnium Project, a framework allowing distanced partners to engage in active and reflective modes of creative dialogue. Yet, Adamczyk and Twidale [2] have argued that multidisciplinary teamwork

\footnotetext{
${ }^{1}$ Sequence of numeric codes used in the broadcast and film industries for synchronizing audio and video material.
} 
has an intricate set of needs, assumptions and requirements that have not been readily met by existing collaborative tools. Fussell et al. [8], who have demonstrated the importance of shared visual context in collaborative work, have also questioned the adequacy of recent video communication technology.

Notable efforts have been undertaken in academia to support creativity and collaboration in music. Works by Jordà \& Barbosa [11] have developed Internet collaborative virtual environments for music applications. They put a special emphasis on performance, composition and production of music by groups of geographically dispersed communities of users, both in synchronous and asynchronous modes. Abrams et al. [1] have also investigated film composers' cognitive process, resulting in the development of a prototype: QSketcher. It offered a flexible workspace to assist composers in their creative workflow by capturing, organizing and manipulating musical ideas. Similarly, Coughlan \& Johnson [7] have designed Sonic Sketchpad, which explored computer support for sketching and representing ideas in collaborative music settings.

Moreover, support for the annotation of artifacts is widely regarded as necessary yet challenging in the collaborative process. Several solutions for text-based documents have already been widely used (Microsoft Office ${ }^{2}$, Google Docs ${ }^{3}$ or Adobe Acrobat ${ }^{4}$ ). A range of tools like Scribblr [18] have also been designed to annotate drawings and image sketches online. Likewise, compelling systems have been proposed for the annotation of multimedia documents and video material [5] [15]. There also exist popular online tools like Viddler $^{5}$ through which users can post comments (textual or video-recorded via a webcam), at particular points in time in a video (Figure 1).

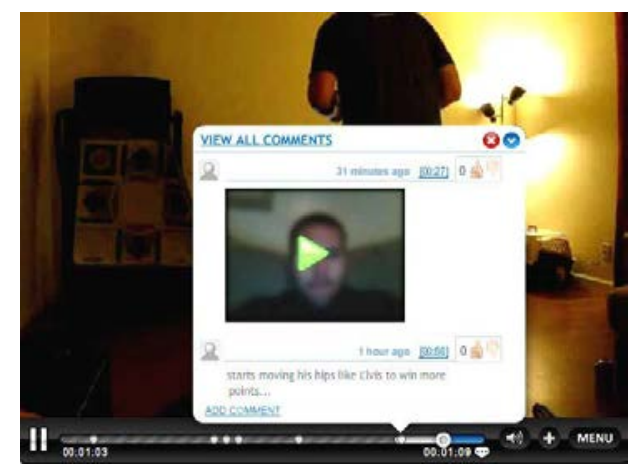

Figure 1. Viddler.com allows users to annotate videos at particular points in time with textual or video comments.

Lastly, a set of Web-based applications dedicated to collaborative music making has recently emerged. Good

\footnotetext{
2 http://www.microsoft.com/office/

${ }^{3}$ http://docs.google.com/

${ }^{4}$ http://www.adobe.com/products/acrobat/

${ }^{5}$ http://www.viddler.com/
}

examples are IndabaMusic.com (Figure 2), Jamglue.com or SpliceMusic.com, through which users can exchange music samples, lead discussions via chatting, forum or in-song commenting tools, and execute multiple editing tasks in a sequencer to compose new pieces.

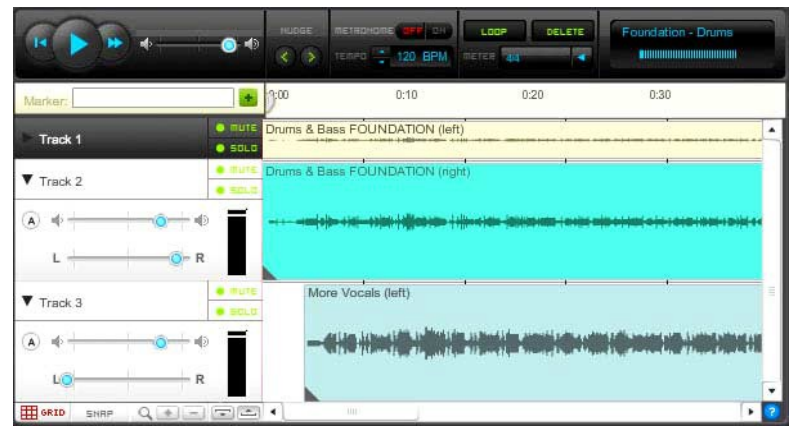

Figure 2. Example of an online sequencer at Indabamusic.com

\section{Our Research}

While technology has been developed to assist professionals in their individual practice (for example video editing tools for filmmakers or virtual instruments and music sequencers for composers), there has been relatively little research done to support the communicative and creative aspects of the composer-filmmaker collaboration. Even if the endeavors or products enumerated above provide promising solutions in their respective niche of activity, there is currently no integrated environment that efficiently facilitates distant communication between composers and filmmakers. Our research is intended to fill this gap by conceiving a system that enables practitioners of the film industry to remotely and asynchronously discuss musical ideas, and in such a way that every stakeholder can clearly and precisely know what is being discussed. Our research follows a process in phases (Figure 3). As mentioned earlier, field studies [13] [14] (Phase 0) originally informed on the problems encountered in the film scoring practice. This led us to two consecutive phases: the design study of a low-fidelity prototype (Phase 1) and the evaluation study of a highfidelity prototype (Phase 2).

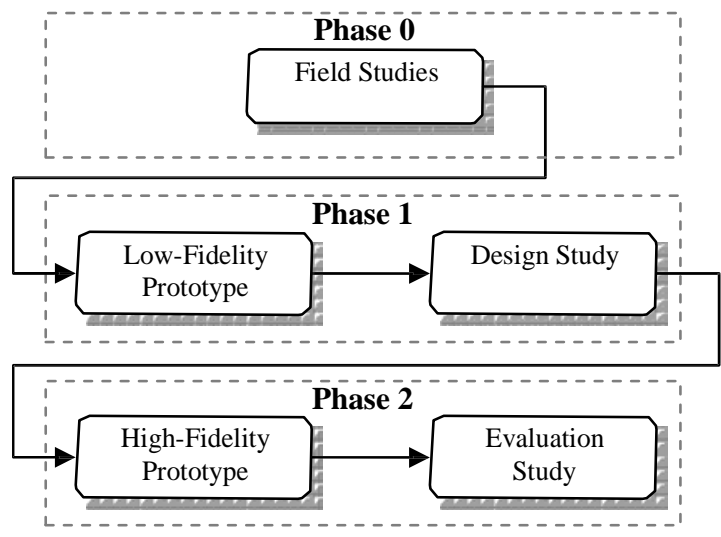

Figure 3. Phases of our research process 
In the following sections the characteristic of phases 1 and 2 will be presented in more detail.

\section{PHASE 1: LOW-FIDELITY PROTOTYPE AND DESIGN STUDY}

\section{Low-Fidelity Prototype}

We developed a Web-based system that allowed users to upload and annotate music or video drafts. The system's most prominent and complex component was the sequencer. We completed the first iteration of the sequencer's design and assembled concepts learned from previous field research into a low-fidelity, paper-based, prototype (Figure 4 and Figure 5). Like most existing sequencers, the prototype enabled basic tasks such as the mixing, editing, syncing and playback of video and music elements. Essentially, users could upload files (e.g. music drafts or video files for particular scenes of the film), import them into the sequencer and position them so that they play back in sync within the mix. This laid a common base of information that every stakeholder could access and refer to. The fact that all parties were then able to visualize and modify the various elements within the same environment constituted the first necessary step in defining a precise and shared scope for communication. In addition, the prototype offered the ability to annotate music samples and video footage present in the mix. Thus, we introduced into the interface the novel concept of discussion tracks (-ein Figure 4).

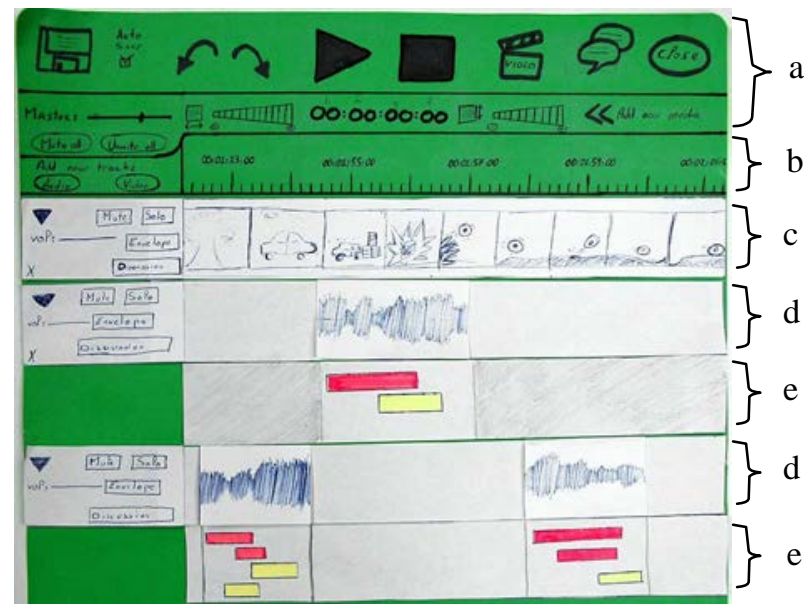

Figure 4. Prototype sequencer overview: (a) Controls,

(b) Timeline, (c) Video track, (d) Audio tracks, (e) Discussion tracks.

The principal novelty of discussion tracks, compared to other tools such as Indaba or Viddler, was in their visual, fully-integrated, representation. Discussion tracks were placed under audio and video tracks and contained stacks of discussion threads. Each thread was symbolized by a small horizontal bar that could be created by clicking and selecting a region within the discussion track. The position and length of each bar respectively represented the start time and duration of the audio or video section above it. This representation enabled the demarcation of specific sections of the mix, therefore establishing a clear temporal scope for each discussion. Clicking on one of the bars opened a window with all comments previously posted in the corresponding thread. It was then possible to post new comments to contribute to the discussion (Figure 5).

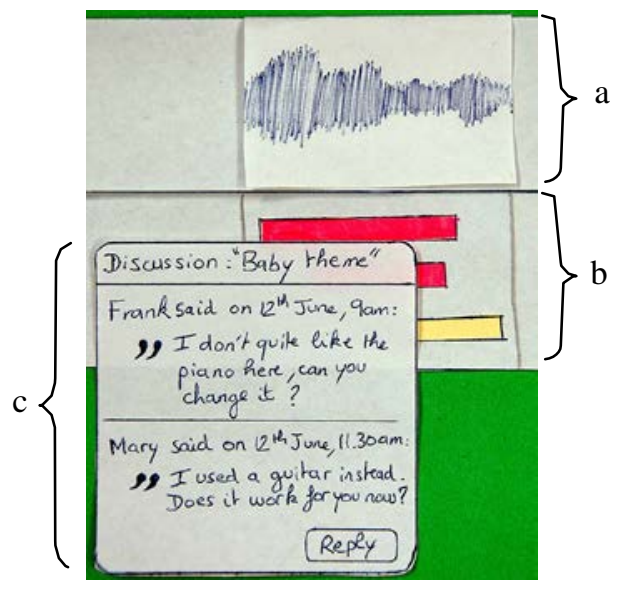

Figure 5. Prototype annotation and discussion system: (a) Audio track containing the waveform of an audio sample, (b) Discussion track containing three threads, (c) Discussion window.

Lastly, whenever changes were made in the mix (e.g. new music samples were added or new comments were posted), all stakeholders were automatically notified with an email sent by the system.

\section{Design Study}

Objectives

We conducted a design study to verify that the prototype was addressing real issues and providing appropriate solutions. Our primary objective was to assess the prototype's usefulness: would it alleviate communication challenges faced by practitioners in remote settings and would practitioners be able to perceive the benefits? At this stage we were mainly concerned with appraising the design principles rather than with testing the usability of the interface. As Greenberg \& Buxton [10] argued, conducting usability tests too early in the design process would have little impact and may even be counterproductive. This was additional motivation for using a paper-based prototype, as it was known to increase chances for study participants to focus on the general characteristics and functions of the system rather than on the aesthetics and low-level interaction details [6].

\section{Participants and Procedure}

The system design still being in its inception we chose to conduct a qualitative and in-depth study with a small group of participants. Three practitioners were recruited (two composers and one director/producer) each of whom had more than 15 years of experience in the film industry and had been working on a wide range of projects throughout their career: documentaries, feature films, short films and advertising. Three individual sessions were organised, each 
session involving one participant and two observers (one leading the experiment, the other one taking notes). The sessions took place at the participants' studios so they could easily refer to their own tools while giving feedback (Figure 6).

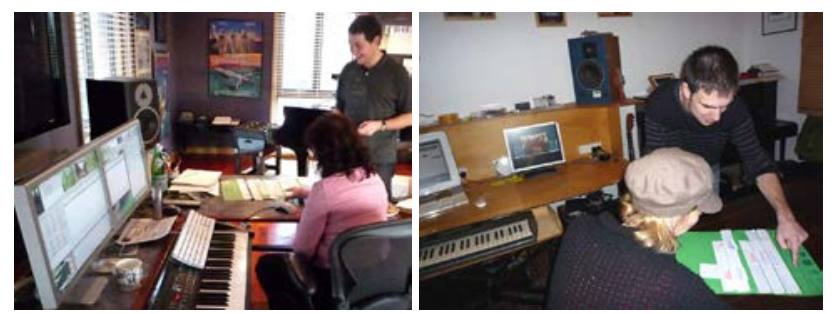

Figure 6. Design study sessions at the composers' studios

Each session lasted for two to three hours where the same procedure was consistently applied. The process began with a demonstration of the system's implemented features: file-sharing tools, portfolios, and event management. Then, a demonstration was given of the prototype sequencer's features: track creation, audio/video editing, and annotation and discussion systems. A scenario walk-through then simulated the various actions directly onto the paper prototype. The scenario, which narrated the fictional story of a Sydney-based composer and a Los Angeles-based director, aimed to explain plausible conditions of use for the system. After watching the demonstrations the participants were asked to specifically comment on the prototype's features. This led to a free discussion where participants gave their opinion on the prototype, shared personal anecdotes, and provided feedback for improvement. Notes of the participants' comments were recorded and further questions were asked probing into particular ideas and issues that were raised in the discussions. This qualitative and flexible method allowed for the collection of rich and contextual data. In the next section we present initial findings that were compiled after the design study.

\section{Initial Findings}

Virtues of Asynchronous Communication

One of our previous field studies [14] had shown that faceto-face meetings were fundamental in building propitious conditions for successful collaboration. Nonetheless, participants of this design study conceded that, in particular instances, they preferred remote and asynchronous modes of communication. On one side, the composers said that they were sometimes frustrated by their clients' feedback and criticisms and that dealing with that frustration was not easy if the clients were standing in the same room. One of them said: "Face-to-face can be very confronting, especially when you deal with 'difficult' people". Being employed by the filmmakers and therefore being responsible for the work, the composers would have to put up with the frustration and behave as if they were in total control of the situation. Hence, the composers reacted positively to the prototype, one of them calling it "a sanctuary, a place of safety". Participants also noted that the system would provide filmmakers with the ability to access at home and listen to the drafts multiple times; whereas during face-to-face meetings they would generally not have enough time to listen to the drafts more than two or three times. On the other side, the director declared she often felt nervous before meeting with composers. She was afraid of hurting their feelings:

"Often my initial reaction is very critical, I think it's more honest that way. But I am nervous when I listen to the music for the first time because I fear I'd have a bad reaction. If the composer sits next to me it could be awkward because they'd see your bad reaction. I guess it's hard for them to take all that criticism because they've probably been working hard on it."

She also said that she would prefer listening to the drafts before the meetings, so she would have the time to ponder over her feelings and would be able to deliver more constructive feedback. Clearly, there is a high level of emotion and sensitivity occurring in creative collaboration between filmmakers and composers, and as evidenced by the participants' testimonies some virtue can be found in asynchronicity. These observations highlight the need for more support regarding the interpersonal communication issues and corroborate the design principles put forward in the prototype.

\section{Scope and Shared Environment}

Mamykina et al. [12] stressed the need for creative professionals to explore ideas within the same environment. Yet, there is currently no integrated environment that efficiently supports distant collaboration between practitioners in the film industry. The participants' feedback especially revealed the lack of coordination between the existing tools they used (eg. email, telephone, instant messaging, or file sharing via File Transfer Protocol (FTP) programs), which was recognized both as a burden and as a source of ambiguity. On the one hand, the director disliked using FTP as it required her to download each individual draft posted by the composer. She also had to manually lay all the drafts in her own editing software before syncing them to the picture. She found it was a lengthy and tedious process, and she appreciated the fact that with our system every element would be centralized and manageable from one place. On the other hand, current communication tools were blamed for potentially carrying ambiguous information, as illustrated by a composer's anecdote:

"I once received some feedback from a director. It was a very long email telling me what he thought about various cues I'd done. He must have spent 3 hours writing that email, and I didn't even quite understand what he was referring to. I wished he could have pointed precisely where the problems were."

Consequently, the annotation feature was well received by the participants. They clearly valued that it would assist 
with framing the scope for discussions, therefore reducing the chance of ambiguity occurring and avoiding time wasted in tedious and uninteresting descriptive tasks.

Participants also made the remark that the system could facilitate the inclusion of the sound designer and film editor in the creative loop. If those third-party collaborators had access to the system they could follow the evolution of the work and contribute to the discussions when necessary. For example the sound designer may advise the composer of all the sound effects that would be laid in the film earlier in the creative process, which would prevent potential conflicts between the music and sound tracks. Also, as noted by the director, a shared environment would help bridge the gap caused by the variety of technologies people were currently using:

"We never got around the problem between the composer and the sound designer. My sound designer works with Protools and my composer with Cubase and there's no way to easily transport the work other than manually.”

These results put an emphasis on the need for implementing a shared collaborative environment and for defining a clear scope for creative discussions.

\section{Work Load Division}

When designing the prototype we were particularly concerned with how the work load performed on the system would practically be divided between the targeted groups of users (filmmakers and composers). Therefore the participants were asked whom they thought would spend more time administering the system, that is, setting up and maintaining the projects, uploading files, creating mixes via the sequencer, managing events and so forth. The answer was unanimous: it would be the composers. On one side, the director assumed that composers would be responsible for most of the work executed on the website. She said that she would probably not have enough time available except for listening and commenting musical pieces, or for doing slight mixing adjustments. On the other side, both composers acknowledged filmmakers' lack of time and both also anticipated taking on the bulk of the work. However, the composers said that it would not necessarily represent a major surplus of work as they were already used to making pre-mixes and to packaging mockups to show their clients. Nonetheless, composers indicated that they would be averse to "doing things twice", that is, making fine-grained mock-ups locally on their computer and then again online on the collaborative system. Practitioners were already used to performing complex mixing and editing tasks with powerful tools on their desktop computers. Hence, they declared they would rather use the prototype system to post pre-mixed elements and then only perform basic mixing tasks on it to illustrate the creative discussions. These remarks thus accentuated the need for supporting the high level collaborative tasks more than the purely technical ones.

\section{PHASE 2: HIGH-FIDELITY PROTOTYPE AND EVALUATION STUDY \\ High-Fidelity Prototype}

Following the design study, a high-fidelity version of the prototype was implemented to give more concrete expression to the design principles. This version was developed as a Web platform consisting of two main components: the general website and the sequencer. The former was implemented using traditional Web technologies: HTML, Javascript, Python, and databases. It allowed basic project management tasks such as creating projects, inviting other users to participate in a project and uploading files or managing events. The latter (illustrated in Figures 7 and 8) was implemented in Flex ${ }^{6}$, a technology permitting the construction of highly interactive Web interfaces, and allowed the creation and precise annotation of music and video mixes.

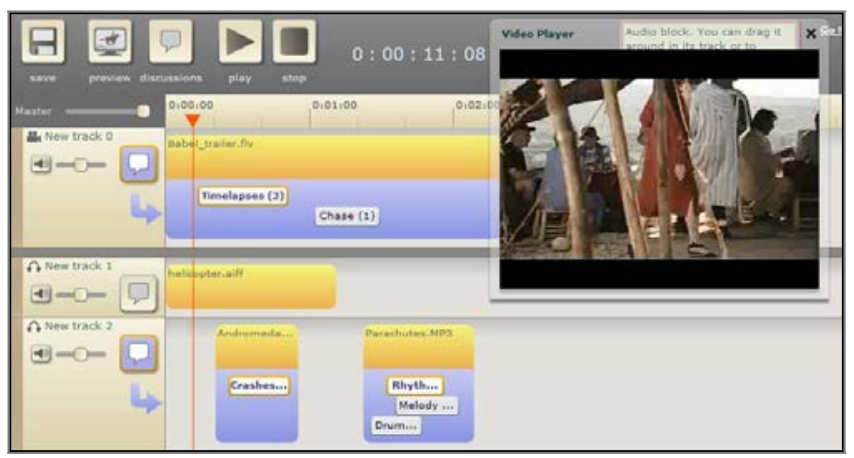

Figure 7. High-fidelity prototype: Sequencer (overview)

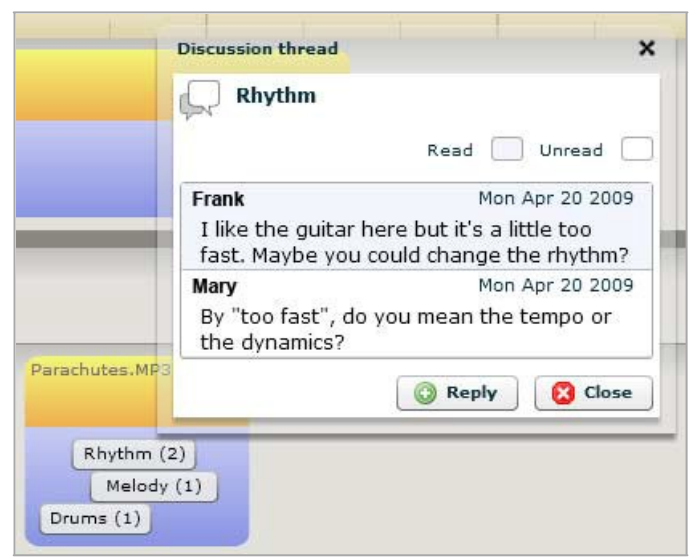

Figure 8. High-fidelity prototype: Discussion blocks and discussion window (close-up)

Moreover, as specified earlier, every file uploaded to the system was automatically encoded on the server with lossy compression algorithms: MP3 for audio files and Flash Video (FLV) for video files. At the expense of sound and visual quality, this process enabled the reduction of file sizes with a 1:5 ratio, thus permitting faster loading via Internet connections. It also allowed the utilization of

\footnotetext{
${ }^{6}$ http://www.adobe.com/products/flex/
} 
formats that could easily be imported into the sequencer. Finally, the prototype rendered asynchronous collaboration possible so that each user could independently annotate and make changes to a mix from their own personal computers. By saving the mix, all changes would be stored in the database and an email notification would automatically be sent to all members of the project so that they could, in turn, open the mix, view the changes and respond to the new comments.

\section{Evaluation Study}

\section{Objectives}

A qualitative evaluation study was organized in the context of a real expert collaboration. The aim was to verify the new assumptions that had emerged from the design study, namely that asynchronous communication was acceptable and that a shared collaborative environment and a precise annotation tool were appropriate for solving indexical issues. At this stage, again, we were more concerned with evaluating the usefulness of the tool rather than its usability. Despite the encouraging findings from the design study and despite the advanced state and robustness of this high-fidelity prototype, real-use testing was still required.

\section{Context and Participants}

Two participants were recruited for the evaluation: one composer and one director. Both were award-winning practitioners with extensive experience in face-to-face and remote collaborations. The composer was based in Sydney, Australia, and the director was based in Perth although he regularly travelled for work to various parts of Australia, such as Alice Springs and Darwin. The composer was already familiar with the research and with the prototype's design principles as she had previously been one of the design study participants. The director, however, was new to this research and did not initially know about the prototype's characteristics and features. This situation helped recreate conditions that were plausible in light of the design study findings, namely that composers would generally be responsible for the work executed on the system and that they would, therefore, naturally become the expert users in this online collaboration.

The practitioners had known each other for eight years and had collaborated on several successful projects: documentaries, feature films and promotional trailers. Because of their geographical separation, they had learned to collaborate remotely and their working methods had evolved over the years with relevant technological advances. At this stage they were used to communicating via email and telephone and to exchanging files via FTP.

For this evaluation, the participants offered to use the prototype in one of their real projects. The selected project was a 50-minute long documentary about the cultural site of Uluru located in central Australia. The project had started three years before when a preliminary version, aimed for preview at festivals, was realized. The composer had already written the entire film score for that version.
But the director later shot additional scenes and required that parts of the music be modified to reflect his updated vision. Therefore, the aim of the work for this evaluation was to discuss and rewrite those cues to produce the final score.

\section{Procedure}

The duration of the evaluation spanned four weeks and all collaboration was conducted remotely. Where we did not meet the director in person, we met the composer once for a one-hour training session at the beginning of the procedure. A similar training session occurred with the director over the phone. The principal reasons for this training were to present the full range of features that the system offered and to limit the influence of potential usability flaws. Data were collected in different ways. First, the participants carbon-copied us to all their email conversations; thus we could follow their collaboration and also observe when the use of email was preferred over that of our system. Second, the composer maintained a log for the phone conversations she had with the director, recording the date, the initiator of the call and a brief summary of the conversation's content; the log was a useful medium to capture conversations that we would not have been able to witness directly. Third, we were automatically notified every time one of the participants executed an action on the system (e.g. after creating or saving a mix). Fourth, the participants filled out a questionnaire at the end of the evaluation giving us feedback on their experience while using the system. The results of the evaluation are presented in the next section.

\section{Results}

Benefits of Precise Annotating

As discussed earlier, the original problem the prototype intended to solve was the ambiguity of scope caused by indexical issues in remote collaboration. In that regard, the novel concept of discussion blocks and threads, enabling precise demarcation and annotation of specific parts of the mix, proved to be useful to the evaluation participants. The composer particularly appreciated this system as it would keep the discussions short. The director also recognized how it would simplify conversations:

"Probably what's most useful is being able to link specific comments to precise locations (e.g "cello seems a bit too prominent here”). There's no doubt about where you're referring to. It's relatively easy to place comments in position with the music. [..] We don't have to say 'At such and such time code could you add some violin?'. It's all visually represented.”

Nonetheless, the evaluation revealed other unexpected benefits for the annotation system. First, it was seen by the participants as a way of showing and reviewing multiple versions of a mix. Second, the composer valued the fact that all comments logged into the system would render clients accountable for their requests; as she said: "It forces the client to be very clear, revealing how vague or 
contradictory they can be, and also keeps an inventory of their demands. It also reveals the delays they cause. This accountability is a good thing”. And third, the director considered that the system would naturally foster more thorough communication: "[w]riting comments down also provides a useful discipline and can help ensure that a director has said everything they need to”.

\section{Asynchronicity + Portability $=$ Flexibility}

The evaluation participants enjoyed the flexibility brought by the system into their workflow, particularly thanks to the prototype's asynchronous and portable characteristics. Initially, the composer believed that asynchronicity would help gain flexibility in time management, arguing that the work could be reviewed at times that are individually convenient rather than scheduled and mutually accessible. She thought that our system would help with the fact that practitioners often work outside the hours where face-toface is appropriate: "The director could look at the mix at $11 \mathrm{pm}$, it doesn't matter [..] It also means that clients can review the work after hours - I have a producer who loves to work very early in the morning, and a director who shoots other productions during post-production”. Indeed, it was observed during this evaluation that most of the email correspondence and most of the communication done via the prototype by the participants occurred in the evenings after 8pm. Even if this observation cannot be generalized, it is necessary for communication systems to cater for these types of situations.

The composer also envisaged that the system would be most beneficial for asynchronously and iteratively reviewing small chunks of the work. In face-to-face situations practitioners tend to present a large volume of work at once to get the most out of their time together. With our system, the composer saw that the work could be broken down into manageable parts and that the downtime spent waiting for each other's replies could be utilized for thinking and exploring new ideas.

Moreover, portability was found to be a key asset of the system. On the one hand, the technologies used for building the prototype were portable in the sense that the system could virtually be used on any common platform or Web browser. For example, while the two participants had two different browsers installed on their computers, they could still both fully use all the features offered by the system. On the other hand, our system was judged portable as it could be used in mobile situations. This was experienced by the director during the evaluation when he once travelled to isolated parts of central Australia. He was then able to connect to the website due to the wireless Internet connection on his laptop and view a mix posted by the composer. The composer also enjoyed this portability as she could access the system from multiple locations outside her studio:

"One of the good unexpected features is the convenience that I too can work away from my studio and access the site from a laptop rather than my main studio. I can take mp3 files and notes and update the files without having to be at the [studio's] computer"

Assuredly, we also believe that the flexibility generated by Web technologies, both in terms of portability and asynchronicity, can favor the establishment of a shared environment between practitioners with diverse backgrounds, habits and workflows.

\section{Limitations of Asynchronous Communication}

Initial findings from the design study had shown that, in some situations, practitioners enjoy asynchronicity because it gives them time to ponder over their feelings and to formulate detailed feedback. However, the director in the evaluation expressed some concerns about asynchronous communication. In particular, although he recognized that getting one's comments in writing is a good discipline for directors who are musically literate, he thought that it can be confronting and limiting for those who do not fully comprehend musical terms:

"Disadvantages would be that the written word can seem quite harsh and also that directors unfamiliar with musical language might find it difficult to express what they're feeling or what they want."

The director generally did not feel comfortable writing criticisms about the composer's music via our system or via email; he was afraid that his words could be too direct or too dry and thus may hurt the composer. For him, synchronous modes of communication, like telephone for example, are often more appropriate for discussing potentially critical matters, as "the instant feedback of even a phone conversation makes it easier to ensure that neither party gets upset with what's said in writing”. Evidently, sensitive conversations can be difficult to handle asynchronously. These issues did not eventually play a significant part during this evaluation because the participants knew each other well and were used to collaborating together creatively. Further investigation with practitioners involved in new working relationships would be necessary to obtain deeper understanding of this delicate topic.

\section{Coordination Issues}

Over the four-week duration of the evaluation, the participants' use of the prototype followed an irregular pattern. The participants sometimes exchanged multiple comments within a few minutes and other times they did not connect to the website for a few days. Some of the long time intervals between connections were justified, for example, by the fact that the composer was busy composing new pieces or that the director was away travelling for work. However, some time intervals were unnecessarily wasted because of a lack of coordination.

As presented earlier, we had originally anticipated coordination issues by implementing an automatic notification system in the prototype. Essentially, an email 
was automatically sent to all members of a project every time someone saved modifications into a mix (e.g. after adding audio/video samples or writing comments). On the one hand, participants found that the automatic notifications were valuable as they enabled awareness of the other's activity and of the availability of new mixes or comments. But on the other hand, this system was not enough to guarantee effective coordination as it did not allow clear identification of what procedure to follow. Although the prototype featured a color scheme to signal unread comments, it was sometimes unclear to the participants who had to take the next action, and when. This resulted, for example, in the composer once waiting for the director's review of a music draft that she had uploaded, while the director was in fact waiting for the composer to provide further drafts. To get around this kind of issue, participants periodically resorted to sending emails or to having phone calls to clarify what they expected each other to do for proceeding with the work.

This showed that more support should be provided to accelerate asynchronous communication and to allow users to delineate more distinctly the expected procedure of the collaboration. For example, a dedicated apparatus could be envisaged to let users define a roadmap for the collaboration and maintain a journal of tasks attached to certain deadlines (e.g. "Review this draft by this date" or "Select your favorite draft and comment on changes to be incorporated before delivery date”). Finally, the composer required the comments be color-coded to reflect their level of urgency and their order of importance. Such prioritization and scheduling functionalities would, according to the participants, save stress and time, both at professional and personal levels.

In the next section a higher perspective is taken to confront and reflect on the findings generated by the two studies presented in this paper.

\section{REFLECTION}

As it is often the case when doing research, our work raised more questions than it brought answers. On the one hand, the design principles proved to be effective addressing the original premise, i.e. resolving the indexical issues and the ambiguity of scope occurring in remote settings; all the study participants considered that the prototype was a useful tool to simplify and clarify creative discussions. On the other hand, the two studies described in this paper unexpectedly raised a number of issues inherent to remote and asynchronous communication in creative practice.

First of all, the requirement for a unified and shared environment was clearly expressed. It also appeared necessary for this environment to be accessible not only by the users originally targeted (i.e. composers, directors and producers), but also by a wider circle of practitioners such as sound designers, music editors and film editors. The studies also confirmed that composers and filmmakers were generally familiar with sequencers as they already used similar tools respectively to produce music and to edit films. Little explanation was required for the participants to understand the system's basic features so this validated the sequencer as a promising candidate for building such a shared and accessible environment.

Second of all, the studies highlighted important advantages and disadvantages of asynchronicity, at multiple levels of collaboration. At the interpersonal level, synchronous communication (particularly face-to-face) was found to be potentially confronting. As revealed in the design study, composers recognized that it could be difficult taking criticisms in person and so our asynchronous system was praised for providing "a sanctuary, a place of safety". The director from the same study also dreaded face-to-face meetings with composers as she feared she could have spontaneous reactions resulting in hurt feelings. However, the evaluation study showed that, in a real-use context, it could also be difficult to give considerate criticisms without the help of synchronous means like face-to-face or telephone. Therefore, a balance should be attained in the use of asynchronous and synchronous communication so as to diffuse emotions and avoid interpersonal clashes. This is a complex problem that depends on a multiplicity of variable parameters, for example: the type of project, the prior personal and professional relationships between collaborators, or the personalities and current moods of the people involved. In that regard, further studies ought to be conducted over long periods of time to evaluate how practitioners adapt and how they reach a comfortable balance between various means of communication. At the coordination level, asynchronicity appeared to be limiting and responsible for provoking unnecessary wait times. This stressed the need for more support in coordinating the collaborative process in remote settings.

Finally, although asynchronicity and Web technologies' portability provided a beneficial flexibility for remote creative collaboration, the evaluation showed that all communications cannot effectively be conveyed by just one type of tool. A wide palette of different tools should be offered to practitioners, who should then be allowed to select the most appropriate tools given their personal taste and the specific context of the collaboration.

\section{CONCLUSION AND FUTURE WORK}

In this paper, we described a system designed to support online and asynchronous collaboration between stakeholders of the film music industry. We then reported on the design study of a paper-based prototype and on the qualitative evaluation of a high-fidelity prototype conducted with filmmakers and composers. This work confirmed the need for further support in resolving ambiguities that occur in distant communication. It also generated a new understanding of the composer-filmmaker relationship and uncovered complex interpersonal and coordination issues that need to be considered in the development of computer tools. While this work validated 
the usefulness of the prototype system and its design principles, more research is required concerning usability issues. Hence, an intense development phase will next be pursued to produce a full-scale working prototype. The usability of the next prototype will be tested with practitioners to ensure that it can effectively be used with minimal or no training. Also, while our system is currently focused on facilitating the remote exchange of information and on clearing the ambiguity of scope, problems related to the ambiguity of content still remain. More work is therefore needed to specifically support an accurate interpretation of the information conveyed via our system. Lastly, over the longer-term, we envisage extending the research to other industries that share similar creative and collaborative dispositions with film scoring, such as architecture, graphic design, dance or dramatic arts.

\section{ACKNOWLEDGEMENTS}

We are extremely grateful to the participants of this study as well as all the composers and filmmakers who have graciously contributed to this research. This research was partly conducted within the Australasian CRC for Interaction Design, which is established and supported under the Australian Government's Cooperative Research Centres Programme. We also wish to express our deep appreciation to the Regional Council of La Réunion (France), the University of La Réunion and the European Union for financial support and helpful encouragement. Finally, our thanks go to Linda Candy, Deborah Turnbull and the anonymous reviewers for their revisions and insightful comments.

\section{REFERENCES}

1. Abrams, S., Bellofatto, R., Fuhrer, R., Oppenheim, D., Wright, J., Boulanger, R., Leonard, N., Mash, D., Rendish, M. \& Smith, J. (2002), 'QSketcher: An Environment for Composing Music for Film', Creativity \& Cognition

2. Adamczyk, P.D. \& Twidale, M.B. (2007), 'Supporting Multidisciplinary Collaboration: Requirements from Novel HCI Education', CHI.

3. Bennett, R. \& Dziekan, V. (2005), 'The Omnium Project - Forming online communities of students, educators and professionals to explore collaborative modes of creative interaction and practice.', 8th International Electronic Theses and Dissertations.

4. Bødker, S., Nielsen, C. \& Petersen, M.G. (2000), 'Creativity, cooperation and interactive design', Proceedings of the conference on Designing interactive systems: processes, practices, methods, and techniques.

5. Bouvin, N.O., Zellweger, P.T., Grønbæk, K. \& Mackinlay, J.D. (2002), 'Fluid annotations through open hypermedia: using and extending emerging web standards', Proceedings of the 11th international conference on World Wide Web.

6. Buxton, B. (2007), Sketching User Experiences, Elsevier.

7. Coughlan, T. \& Johnson, P. (2006), 'Interaction in creative tasks: Ideation, Representation and Evaluation in Composition', SIGCHI conference on Human Factors in computing systems.

8. Fussell, S.R., Kraut, R.E. \& Siegel, J. (2000), 'Coordination of communication: effects of shared visual context on collaborative work', Proceedings of the 2000 ACM conference on Computer supported cooperative work.

9. Gaver, W.W., Beaver, J. \& Benford, S. (2003), 'Ambiguity as a Resource for Design', ACM Special Interest Group on Human Computer Interaction (CHI).

10. Greenberg, S. \& Buxton, B. (2008), 'Usability evaluation considered harmful (some of the time)', SIGCHI conference on Human factors in computing systems.

11. Jordà, S. \& Barbosa, Á. (2001), 'Computer Supported Cooperative Music: Overview of Research Work and Projects at the Audiovisual Institute-UPF', Workshop on Current Research Directions in Computer Music,

12. Mamykina, L., Candy, L. \& Edmonds, E. (2002), 'Collaborative creativity', Communications of the ACM, vol. 45 , no. 10

13. Phalip, J. \& Edmonds, E. (2007), 'Guidelines for Communication in Film Scoring', International Conference on Music Communication Science.

14. Phalip, J., Morphett, M. \& Edmonds, E. (2007), 'Alleviating Communication Challenges in Film Scoring: An Interaction Design Approach.', OZCHI 2007, the Australasian Computer-Human Interaction Conference.

15. Ramos, G. \& Balakrishnan, R. (2003), 'Fluid interaction techniques for the control and annotation of digital video', Proceedings of the 16th annual ACM symposium on User interface software and technology.

16. Schober, M.F. (2006), 'Virtual environments for creative work in collaborative music-making', Virtual Reality, vol. 10, no. 2

17. Stacey, M. \& Eckert, C. (2003), 'Against Ambiguity', Computer Supported Cooperative Work, vol. 12, no. 2

18. Weakley, A., Deverell, K. \& Yuille, J. (2007), 'WEB 2.0 in Support of Sketching in Architectural Practice', Semantic Web and Web 2.0 in Architectural, Product, Engineering Design Workshop (SWinDESIGN2007). 